\title{
OPEN Lag synchronization of coupled time-delayed FitzHugh-Nagumo neural networks via feedback control
}

\author{
Malik Muhammad Ibrahim ${ }^{1}$, Muhammad Ahmad Kamran², \\ Malik Muhammad Naeem Mannan ${ }^{3}$, Il Hyo Jung ${ }^{1 \bowtie}$ \& Sangil Kim ${ }^{1 \bowtie}$
}

Synchronization plays a significant role in information transfer and decision-making by neurons and brain neural networks. The development of control strategies for synchronizing a network of chaotic neurons with time delays, different direction-dependent coupling (unidirectional and bidirectional), and noise, particularly under external disturbances, is an essential and very challenging task. Researchers have extensively studied the synchronization mechanism of two coupled time-delayed neurons with bidirectional coupling and without incorporating the effect of noise, but not for timedelayed neural networks. To overcome these limitations, this study investigates the synchronization problem in a network of coupled FitzHugh-Nagumo (FHN) neurons by incorporating time delays, different direction-dependent coupling (unidirectional and bidirectional), noise, and ionic and external disturbances in the mathematical models. More specifically, this study investigates the synchronization of time-delayed unidirectional and bidirectional ring-structured FHN neuronal systems with and without external noise. Different gap junctions and delay parameters are used to incorporate time-delay dynamics in both neuronal networks. We also investigate the influence of the time delays between connected neurons on synchronization conditions. Further, to ensure the synchronization of the time-delayed FHN neuronal networks, different adaptive control laws are proposed for both unidirectional and bidirectional neuronal networks. In addition, necessary and sufficient conditions to achieve synchronization are provided by employing the Lyapunov stability theory. The results of numerical simulations conducted for different-sized multiple networks of timedelayed FHN neurons verify the effectiveness of the proposed adaptive control schemes.

Synchronization plays a tremendous role in information transfer and decision-making in different fields of science and technology ${ }^{1}$. In the past decade, many researchers have investigated different synchronization schemes, methodologies, and regimes for chaotic systems such as lag synchronization ${ }^{2}$, generalized synchronization ${ }^{3}$, phase synchronization $^{4}$, projective synchronization ${ }^{5}$, anticipating synchronization ${ }^{6}$, cluster synchronization ${ }^{7}$, and consecutive synchronization ${ }^{8}$. Lag synchronization, introduced for drive and response systems and extensively studied, is defined as an overlapping of the shifted-in-time states of two systems with a positive constant.

In neuroscience, the interactions of neurons and their networks have been the primary targets for investigating the differences in the functionality and dynamics of healthy and diseased brains ${ }^{9-11}$. For example, the brain responses of stroke patients have been analyzed and compared with those of healthy subjects to develop an effective rehabilitation system ${ }^{12,13}$. Neurons in the brain are connected to other neurons in the same or other regions to form a network of neurons for communication and efficient information processing. In the past decades, the internal dynamics behind the coordination and communication mechanism of neurons and their networks have been explored by developing different strategies and systems ${ }^{14-18}$. The processing of cognitive information in the brain is based upon the synchronized interactions between large numbers of neurons distributed within and across different specialized brain regions ${ }^{19}$. Researchers have found that synchronization between individual neurons or networks of neurons is an important factor for the stable and efficient working of the brain ${ }^{20,21}$.

${ }^{1}$ Department of Mathematics, Pusan National University, Busan 46241, Republic of Korea. ${ }^{2}$ Department of Cogno-Mechatronics Engineering, Pusan National University, Busan 46241, Republic of Korea. ${ }^{3}$ School of Allied Health Sciences, Griffith University, Gold Coast QLD 4222, Australia. ${ }^{\boxplus}$ email: ilhjung@pusan.ac.kr; sangil.kim@ pusan.ac.kr 
Experimental and theoretical results from previous studies suggested that synchronization of neuronal activity is not only a major property of cortical and subcortical networks within and across different brain regions but also helps to perform many functions in cognitive processes ${ }^{19}$. Therefore, synchronization can be considered as the basis for signal transmission and processing in both healthy and abnormal brains ${ }^{20}$. For instance, previous studies found synchronization in the brain regions including hippocampal and olfactory ${ }^{22}$, and it has been reported that brain disorders and disorder in body functionalities such as heart rhythm and gait could be caused by the absence of synchronization between neurons ${ }^{23}$. Furthermore, past research showed that certain brain diseases, such as Alzheimer's disease, epilepsy, Parkinson's, autism, and schizophrenia could be caused due to abnormal/ absence neural synchronization ${ }^{20,22,24-26}$.

The synchronization of brain networks is essential for normal functioning, and understanding its dynamics is important to many aspects of our lives ${ }^{27}$. Research on synchronization is vital in revealing the role of communication cells in the neural network structure ${ }^{28-30}$. These communication cells are coupled by specialized intercellular pathways, which have been identified as gap junctions, and many researchers have been extensively studying the influence of the gap junctions on the synchronization behavior between two or three neuronal systems ${ }^{28,31}$ These $^{2}$ gap junctions, known as protein channels, play an important role in the transmission of information between neurons. Many well-established mathematical models, such as the integrated-fire model $(1907)^{32}$, the HodgkinHuxley model (HH) $(1952)^{33}$, the FHN model (1962), the Morris and Lecar model (1981) $)^{34}$, and the Hind-marsh and Rose model $(1984)^{35}$, have been used to study the synchronization of neurons. Among these mathematical models, the FHN neuron model, developed by Fitzhugh ${ }^{36}$ and Nagumo et al. ${ }^{37}$, has been used as a primary tool for investigating neuronal synchronization problems $s^{20,28,38,39}$. Different schemes, such as the backstepping design $\operatorname{method}^{40}$, adaptive synchronization method ${ }^{41}$, linear and nonlinear feedback synchronization method ${ }^{28}$, sliding mode control method ${ }^{20}$, time-delay feedback approach ${ }^{42}$, and impulsive synchronization method ${ }^{43}$, have been proposed to achieve synchronization in chaotic systems.

On the other hand, time delays are a fundamental part of almost all biological phenomena. The finite propagation speed of the action potential along the axons of neurons and time lapses in information transmission (synaptic process) and reception (dendritic process) between neurons produce time delays in real neurons and their networks ${ }^{44}$. Previous studies have shown that the speed of the action potential propagation could be significantly reduced (as slow as $1 \mathrm{~m} / \mathrm{s}$ ) by unmyelinated axons (myelin is an insulating layer that allows electrical impulses to transmit smoothly and quickly along the neuron) that cause large time delays (as high as $80 \mathrm{~m} / \mathrm{s}$ ) in information processing in neuronal networks ${ }^{45,46}$. Thus, time delays play an important role in the dynamics of neurons and their networks and should be included in mathematical modeling and analysis ${ }^{28,47,48}$. Recently, many researchers have investigated the effects of time delays in neuronal networks and found many delay-induced phenomena ${ }^{49-52}$. For instance, it has been reported that delay-enhanced synchronization may be essential for information transmission in neuronal networks ${ }^{53,54}$. It has also been reported that the firing dynamics of neurons are affected by many factors, such as noise, connection configuration, and coupling delays. For example, a recent study revealed that coupling delays present in the electrical or chemical synaptic connections can influence the synchronization of neuronal firing ${ }^{55,56}$.

Researchers have also studied the synchronization in time-delayed neuronal systems of two coupled neurons. Bin et al. used two coupled FHN neurons to examine the effects of time-delays on the synchronization dynamics between neurons ${ }^{57}$. Jia et al. investigated the influences and effects of time-delays on the dynamics of a coupled FHN neuronal system ${ }^{58}$. Wang et al. studied the synchronization phenomenon in the time-delayed chaotic system with unknown and uncertain parameters and developed an intermittent adaptive control scheme to guarantee synchronization between neurons ${ }^{41}$. Ibrahim and Jung developed an adaptive control methodology to guarantee synchronization between a bidirectional ring-structured FHN neuronal network under the effect of external electrical stimulation with single- and dual-state gap junctions ${ }^{1}$. Zhang investigated the out-lag synchronization by proposing a pinning control scheme in fractional-order complex networks with coupling and internal delays ${ }^{59}$. Petkoski and Jirsa studied how the phase lag synchronization between the neurons of different brain regions is governed by the spatio-temporal organization of the brain by using self-sustained time-delayed chaotic oscillators ${ }^{60}$. Liu and Puming recorded electrocorticogram (ECoG) data from eleven refractory epilepsy patients and utilized the phase synchronization phenomenon to map brain networks ${ }^{61}$. Siddique et al. used a chaotic master-slave system with unknown parameters, bounded delay rates, finite time-delay, and perturbations to investigate the synchronization phenomenon by developing local adaptive and robust adaptive control methodologies ${ }^{62}$. Siddique and Rehan proposed observer-based control strategies to realize synchronization in drive and response chaotic systems ${ }^{63}$. Jia et al. proposed a time-delayed coupled FHN system and studied its stability and Hopf bifurcation ${ }^{58}$. Riaz et al. used a nonlinear drive-response system with time-delay and under the effect of slope restricted input nonlinearity to investigate the synchronization phenomenon ${ }^{64}$. Zaheer et al. addressed the synchronization in non-linear coupled time-delayed systems by developing a novel state feedback delay-range-dependent control scheme ${ }^{65}$. Rehan and Hong studied the synchronization in coupled uncertain time-delayed FHN system with parametric variations under the effect of external electrical stimulation ${ }^{66}$.

Furthermore, the addition of noise in a dynamical system and its effects on the dynamical properties of the system are considered as one of the important research issues in the past decade, which has uncovered phenomena such as stochastic resonance ${ }^{67}$, coherent resonance ${ }^{68}$, and noise-sustained synchronization ${ }^{69}$ in nonlinear dynamical systems. Previous research has shown that neurons adjust their dynamics and firing behavior to transmit information optimally in the presence of noise ${ }^{70}$. Therefore, the addition of noise in time-delayed FHN neurons will make it more realistic but complex and difficult to investigate the synchronization in a network of neurons. However, as discussed above, a coupled system of two or three neurons cannot depict the dynamics of the network of neurons. Furthermore, the synchronization of noisy FHN neurons has not been investigated extensively in past research. Therefore, it is important to investigate and analyze the synchronization properties of a time-delayed network of FHN neurons in the absence and presence of noise. Ultimately, the development 


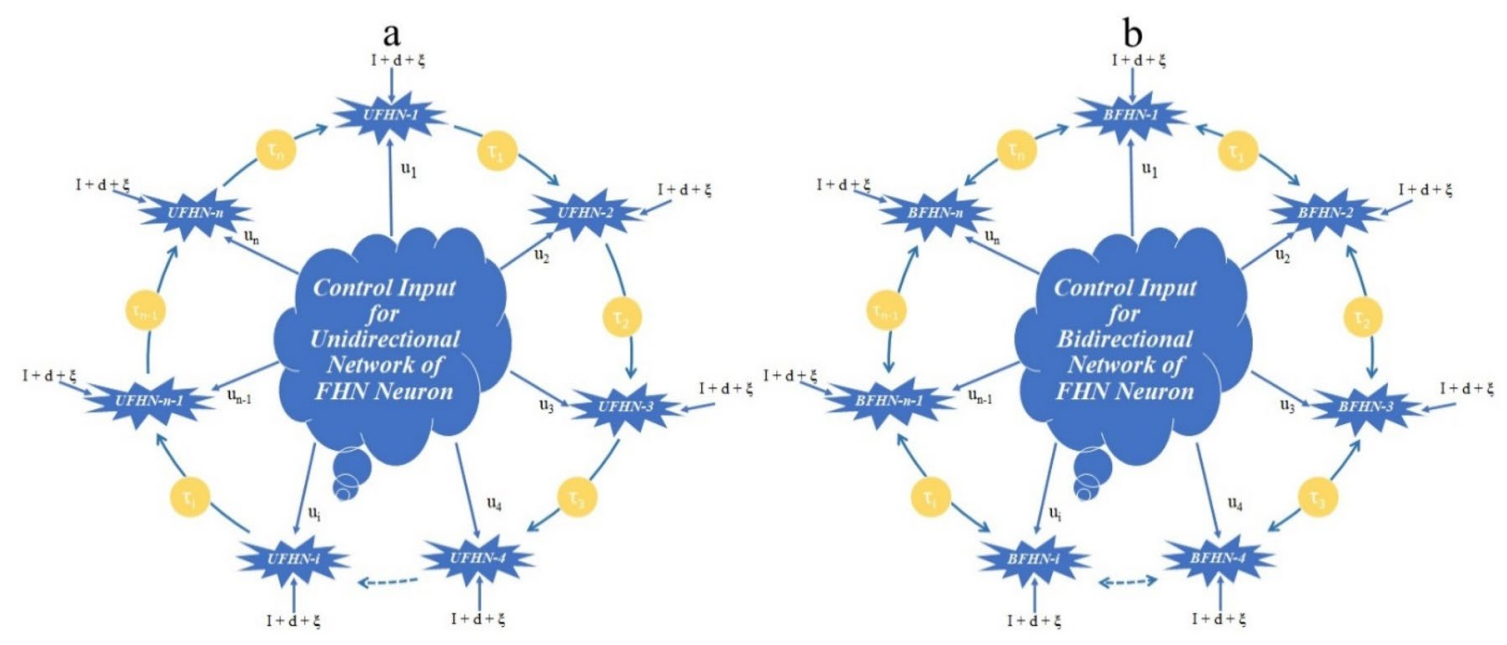

Figure 1. Schematic diagram of ring-structured delayed FHN neuronal networks. (a) Unidirectional coupling. (b) Bidirectional coupling.

of control strategies for synchronization of a network of chaotic neurons with time delays, different directiondepend coupling (unidirectional and bidirectional), and noise, particularly under external disturbances, is very important and challenging.

This paper examines the synchronization phenomena of unidirectional and bidirectional ring-structured FHN networks with and without noise under ionic and EES with different gap junctions and time-delay dynamics between the neurons. Each neuron of the unidirectional network is connected to the next neuron via synapses, and the first neuron of the network is a master neuron for connecting networks. However, each neuron in a bidirectional network is connected to the next neuron as well as the previous neuron behaves as a master and slave neuron simultaneously. The configuration of both networks is shown in Fig. 1a, b. The separation between each neuron in both networks is incorporated through different time-delay parameters.

This paper also investigates the influence of the time delay between connected neurons on synchronization conditions. Unique and different adaptive control laws are proposed for both unidirectional and bidirectional neuronal networks, which guarantee the synchronization of time-delayed FHN networks in the absence and presence of noise. Necessary and sufficient conditions were driven using the Lyapunov theory of stability, which also assures the synchronization of delayed FHN networks. Furthermore, the proposed control schemes were verified using multiple networks consisting of five, ten, fifty, one hundred, two hundred and fifty, five hundred, and one thousand time-delayed FHN neurons with and without noise through numerical simulations.

The main contributions of this paper include (1) investigating the synchronization of a unidirectional network of $n$-FHN neurons in the absence and presence of noise with different gap-junctions and time delays, (2) investigating the synchronization of a bidirectional network of $n$-FHN neurons in the absence and presence of noise with different gap-junctions and time delays, (3) the development of unique and different adaptive control laws for both unidirectional and bidirectional networks, and (4) achieving the synchronization of membrane and recovery states for both unidirectional and bidirectional FHN neurons using the proposed adaptive schemes.

Time-delayed FHN neuronal networks and control design. This section presents the mathematical formulation of the synchronization problem for unidirectional and bidirectional neuronal networks of $n$ timedelayed FHN neurons with and without noise connected in a ring structure, under ionic and EES, and with different time delays and gap junctions. Based on the literature, we hypothesized that both unidirectional and bidirectional time-delayed FHN neuronal networks will show very complex and unpredictable behaviors and dynamics with delays in gap junctions. Furthermore, the inclusion of the noise and dynamic effects of ionic gate disturbances in the network will make it more realistic but more challenging to analyze.

Definition A system of neurons is said to be synchronized if for all initial conditions $x_{i}(0)$ and $y_{i}(0)$, $\lim _{x \rightarrow \infty}\left\|e_{x_{i}}\right\|=0$ and $\lim _{y \rightarrow \infty}\left\|e_{y_{i}}\right\|=0$.

\section{Unidirectional time-delayed FHN neuronal networks.}

\section{(a) Network without noise}

Let us consider a unidirectional ring-structured FHN network with different gap-junctions, time delays, and without any external noise between the synapses of connecting neurons. Mathematically, 


$$
\begin{aligned}
& \dot{x}_{i}=x_{i}\left(x_{i}-1\right)\left(1-r x_{i}\right)-y_{i}-g_{i}\left(x_{i}\left(t-\tau_{i}\right)-x_{i+1}\right)+I+d+u_{i} \\
& \dot{y}_{i}=b x_{i}-c y_{i}
\end{aligned}
$$

where $x_{i}$ and $y_{i}$ are stated action potential and recovery variables, respectively; $r, b$, and $c$ are positive constants; $d=0.01 \sin (0.2 t)$ is the ionic gate disturbance; $I=\frac{A}{\omega} \cos (\omega t)$ is the stimulus current; $g_{i}$ represents the coupling strength of the gap junction between master and slave neurons; $\tau_{i}$ is non-negative delay parameter; $u_{i}$ represents the control; and $i=1,2 \ldots, n$ is the number of FHN neurons.

\section{(b) Network with noise}

Let us consider a unidirectional ring-structured FHN network with different gap-junctions, time delays, and external noise between the synapses of connecting neurons. Mathematically,

$$
\begin{aligned}
& \dot{x}_{i}=x_{i}\left(x_{i}-1\right)\left(1-r x_{i}\right)-y_{i}-g_{i}\left(x_{i}\left(t-\tau_{i}\right)-x_{i+1}\right)+I+d+\varphi_{1}+u_{i} \\
& \dot{y}_{i}=b x_{i}-c y_{i}
\end{aligned}
$$

where $\varphi_{1}(t)$ is a Gaussian noise ${ }^{70}$ source having zero mean and correlation function:

$$
\left\langle\varphi_{1}(t) \varphi_{1}\left(t^{\prime}\right)\right\rangle=2 D \delta\left(t-t^{\prime}\right)
$$

\section{(c) Control laws design}

The error states of the systems described by Eqs. (1) and (2) can be defined as

$$
e_{x_{i}}=x_{i}\left(t-\tau_{i}\right)-x_{i+1}, e_{y_{i}}=y_{i}\left(t-\tau_{i}\right)-y_{i+1}
$$

Taking the time derivative of the error system, Eq. (4) can be expressed as shown in Eq. (5).

$$
\begin{aligned}
\dot{e}_{x_{i}}= & -r e_{x_{i}}\left(x_{i}^{2}\left(t-\tau_{i}\right)+x_{i}\left(t-\tau_{i}\right) x_{i+1}+x_{i+1}^{2}\right)+\left(r e_{x_{i}}+e_{x_{i}}\right)\left(x_{i}\left(t-\tau_{i}\right)+x_{i+1}\right)-e_{x_{i}} \\
& -e_{y_{i}}-g_{i}\left(x_{i}\left(t-2 \tau_{i}\right)-x_{i+1}\left(t-\tau_{i}\right)\right)+g_{i+1}\left(x_{i+1}\left(t-\tau_{i+1}\right)-x_{i+2}\right)+u_{i}-u_{i+1} \\
\dot{e}_{y_{i}}= & b\left(x_{i}\left(t-\tau_{i}\right)-x_{i+1}\right)-c e_{y_{i}}
\end{aligned}
$$

Next, we propose a unique control scheme by using Lyapunov stability and adaptive control theories.

Theorem 1 Consider the unidirectional time-delayed FHN systems as described in Eqs. (1) and (2) with the dynamical error system described by Eq. (5). If the controllers $u_{i}$ in the system defined by Eq. (5) are defined as

$$
u_{i}=(b+c)\left[\left(x_{i-1}\left(t-\tau_{i-1}\right)-x_{i}\right)\left(\exp \left(\left(x_{i}\left(t-\tau_{1}\right)\right)+\left(x_{i+1}\left(t-\tau_{1}\right)+1\right)\right)\right)\right],
$$

then the synchronization of unidirectional networks of the time-delayed FHN systems described in Eqs. (1) and (2) can be achieved by converging the error of the synchronized system to zero.

Proof Please see the supplementary information for the proof.

\section{Bidirectional time-delayed FHN neuronal networks.}

\section{(a) Network without noise}

Let us consider a bidirectional ring-structured time-delayed FHN network with different gap junctions and without any external noise. Mathematically,

$$
\begin{aligned}
\dot{x}_{j}= & x_{j}\left(x_{j}-1\right)\left(1-r x_{j}\right)-y_{j}-g_{2 j-1}\left(x_{j}-x_{j-1}\left(t-\tau_{j-1}\right)\right)-g_{2 j}\left(x_{j}\left(t-\tau_{j}\right)-x_{j+1}\right) \\
& +I+d+u_{j} \\
\dot{y}_{j}= & b x_{j}-c y_{j}
\end{aligned}
$$

where $g_{2 j-1}$ and $g_{2 j}$ represent the coupling strength of the gap junction between the master and slave neurons, and $\tau_{j-1}$ and $\tau_{j}$ are the delay parameters between the neurons, for $j=1,2,3, \ldots, n$.

\section{(b) Network with noise}

Let us consider a bidirectional ring-structured time-delayed FHN network with different gap junctions and external noise. Mathematically, 


\begin{tabular}{|l|l|}
\hline Parameter & Value \\
\hline$r$ & 10 \\
\hline$b$ & 1 \\
\hline$c$ & 0.003 \\
\hline$A$ & 0.1 \\
\hline$w$ & $2 \pi f$ \\
\hline$f$ & 0.131 \\
\hline
\end{tabular}

Table 1. Values of the parameters used in the numerical simulations.

$$
\begin{aligned}
\dot{x}_{j}= & x_{j}\left(x_{j}-1\right)\left(1-r x_{j}\right)-y_{j}-g_{2 j-1}\left(x_{j}-x_{j-1}\left(t-\tau_{j-1}\right)\right)-g_{2 j}\left(x_{j}\left(t-\tau_{j}\right)-x_{j+1}\right) \\
& +I+d+\varphi_{2}+u_{j} \\
\dot{y}_{j}= & b x_{j}-c y_{j}
\end{aligned}
$$

where $\varphi_{2}(t)$ is the Gaussian noise $\mathrm{e}^{70}$ source having zero mean and the following correlation function:

$$
\left\langle\varphi_{2}(t) \varphi_{2}\left(t^{\prime}\right)\right\rangle=2 D \delta\left(t-t^{\prime}\right)
$$

\section{(c) Control laws design}

Taking the derivative of Eq. (4) with respect to time, the error system for the time-delayed FHN networks described in Eqs. (6) and (7) can be expressed as shown by Eq. (9).

$$
\begin{aligned}
\dot{e}_{x_{j}}= & -r e_{x_{j}}\left(x_{j}^{2}\left(t-\tau_{j}\right)+x_{j}\left(t-\tau_{j}\right) x_{j+1}+x_{j+1}^{2}\right)+\left(r e_{x_{j}}+e_{x_{j}}\right)\left(x_{j}\left(t-\tau_{j}\right)+x_{j+1}\right)-e_{x_{j}} \\
& -e_{y_{j}}-g_{2 j-1}\left(x_{j}\left(t-\tau_{j}\right)-x_{j-1}\left(t-\tau_{j}-\tau_{j-1}\right)\right)-g_{2 j} e_{x_{j}}\left(t-\tau_{j}\right)+g_{2 j+1}\left(x_{j+1}-x_{j}\left(t-\tau_{i}\right)\right)-g_{2 j+2} e_{x_{j+1}} \\
& +u_{j}-u_{j+1} \\
\dot{e}_{y_{j}}= & b\left(x_{j}\left(t-\tau_{j}\right)-x_{j+1}\right)-c e_{y_{j}}
\end{aligned}
$$

Next, we propose a unique control scheme by using Lyapunov stability and adaptive control theories.

Theorem 2 Consider bidirectional time-delayed FHN networks as described in Eqs. (6) and (7) with the dynamical error system of Eq. (9). If the controllers $u_{j}$ in the error system are defined as

$$
u_{j}=(b+c)\left[\left(x_{j-1}\left(t-\tau_{j-1}\right)-x_{j}\right)\left(\exp \left(\left(x_{j}\left(t-\tau_{1}\right)\right)\left(x_{j+1}\left(t-\tau_{1}\right)+1\right)\right)\right)\right]+\left(x_{j-1}\left(t-\tau_{j-1}\right)-x_{j}\right)
$$

then this will ensure the synchronization of the bidirectional networks of the time-delayed FHN systems described in Eqs. (6) and (7) by converging the error of the synchronized system to zero.

Proof Please see the supplementary information for the proof.

\section{Results}

After creating an accurate model for the dynamics of the ring-structured network of $n$-identical FHN neurons with and without noise, we establish a synchronization control scheme for achieving the coherent behavior of the neurons. Numerical simulations were executed to verify the proposed control laws and examine their impact on the synchronization of time-delayed unidirectional and bidirectional ring-structured FHN networks composed of five, ten, fifty, one hundred, two hundred and fifty, five hundred, and one thousand neurons. The parameter values used in this study are listed in Table 1 . The values for $g, \tau$, and initial conditions are randomly chosen from $(0-0.1),(3-35)$, and $((0-0.5),(0-0.5))$, respectively.

\section{Analysis of unidirectional time-delayed FHN networks.}

\section{(a) Networks without noise}

The proposed unidirectional networks of five, ten, fifty, one hundred, two hundred and fifty, five hundred, and one thousand neurons with different gap-junctions and time delays can be modeled as presented in Eq. (1) with $i=1,2, \ldots, 1000$.

In the case of the unidirectional network without any noise, the unsynchronized error dynamics for the membrane potential states and recovery variable states for the network of five neurons (blue line), ten neurons (red line), fifty neurons (black line), one hundred neurons (green line), two hundred and fifty neurons (magenta line), five hundred neurons (brown line), and one thousand neurons (cyan line) are shown in Figs. 2a and 3a. 
(a)

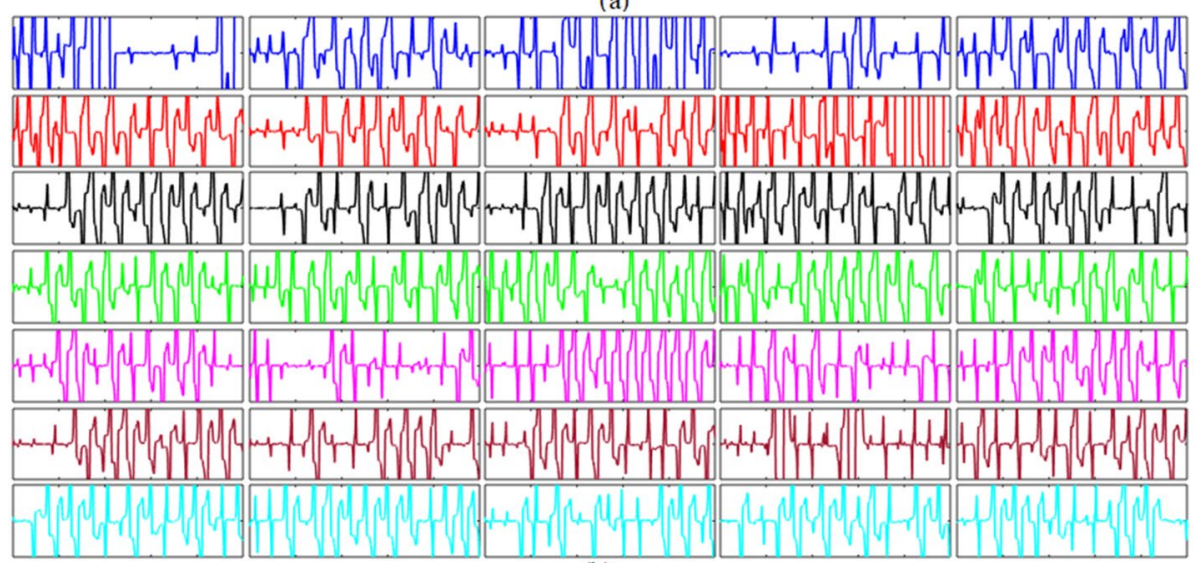

(b)
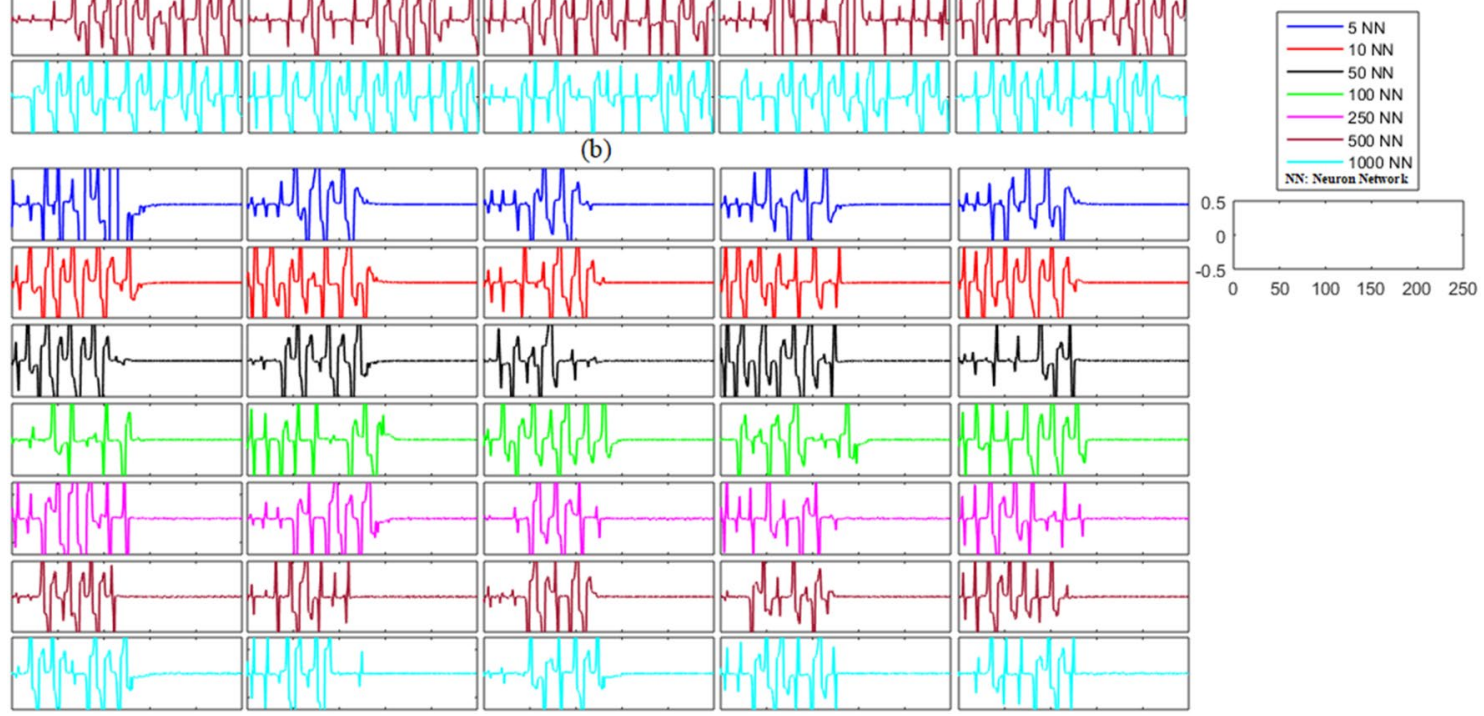

Figure 2. (a) Without control synchronization dynamics of the membrane potential states (x-states) of different time-delayed unidirectional FHN networks without noise. (b) Controlled synchronized error dynamics of the membrane potential states (x-state) of different time-delayed unidirectional FHN networks without noise.

These figures show the error dynamics of five randomly selected pairs of neurons from each network. These plots reveal that the error dynamics are not convergent for both states and the activity of neurons in all networks is highly non-synchronized.

Next, we analyzed the results shown in Figs. $2 \mathrm{~b}$ and $3 \mathrm{~b}$ after the implementation of the proposed control laws for the unidirectional time-delayed network without noise. We analyzed the effectiveness of the proposed control scheme by examining the synchronization dynamics of the ring-structured delayed FHN neuronal network, with and without the adaptive control. The outcomes of this examination are shown in Figs. $2 \mathrm{~b}$ and $3 \mathrm{~b}$. The proposed controllers for the unidirectional network were activated at $t=130$. The results show oscillatory and nonsynchronized behavior for all networks before the application of the proposed controller. In contrast, when the controller was activated at $t=130$, the errors between the membrane potential and recovery variables converged to zero, indicating the synchronization between both states of the delayed unidirectional FHN neuronal networks. Furthermore, we calculated the mean errors for both membrane potential states and recovery variable states in each network to show the overall effectiveness of the proposed control scheme for the non-noisy unidirectional network of time-delayed FHN networks. The results of this analysis are listed in Table 2. It can be visualized that the error for both states in each network is almost zero, indicating the synchronized behavior of the network.

\section{(b) Networks with noise}

Next, we investigated the synchronization problem in unidirectional networks with noise. The results of this analysis are shown in Figs. 4 and 5 and Table 3. The results in Figs. $4 \mathrm{a}$ and $5 \mathrm{a}$ show the unexpected behavior of the neurons in each network and indicate that the primary neuronal networks of delayed unidirectional FHN neurons with noise are not synchronized. It can be concluded at this stage that the non-synchronized behavior is present during the firing of the unidirectional neurons of the delayed networks. The results in Figs. $4 \mathrm{~b}$ and $5 \mathrm{~b}$ illustrate the effectiveness of the proposed control scheme for noisy networks of FHN neurons. Initially, the controller was switched off until $t=130$, and it can be seen that five randomly selected error dynamics show non-zero and non-convergent spikey behavior, indicating that the activity of the neurons in each network is non-synchronized. In contrast, the error dynamics converged to zero and all the networks achieved synchronization as soon as the controller is switched on at $t=130$. Furthermore, the mean errors for each state and each 
(a)

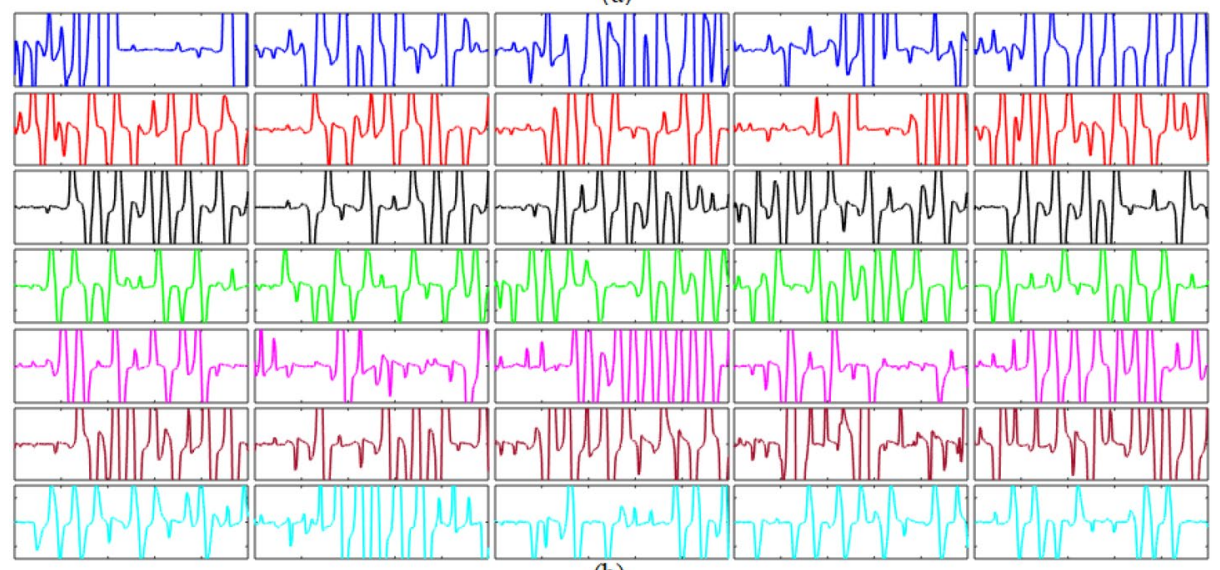

(b)
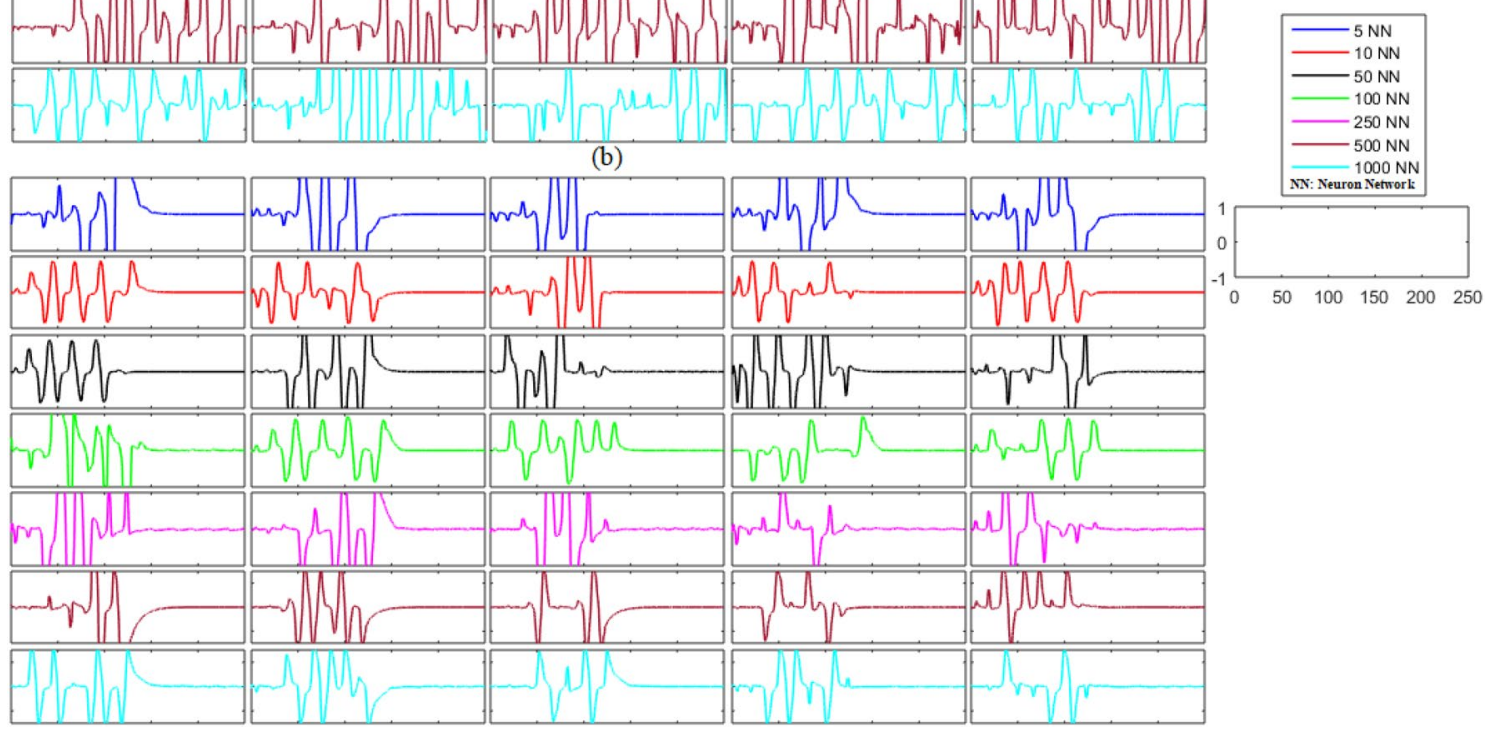

Figure 3. (a) Without control synchronization dynamics of the recovery variable states (y-states) of different time-delayed unidirectional FHN networks without noise. (b) Controlled synchronized error dynamics of the recovery variable states (y-states) of different time-delayed unidirectional FHN neuronal networks without noise.

\begin{tabular}{|l|c|l|}
\hline Number of neurons in network & Membrane potential states & Recovery variable states \\
\hline 5 & $-1.2734 \times 10^{-21}$ & 0 \\
\hline 10 & $-1.0508 \times 10^{-20}$ & $3.0227 \times 10^{-20}$ \\
\hline 50 & $-1.4520 \times 10^{23}$ & $-3.2524 \times 10^{-21}$ \\
\hline 100 & $6.8429 \times 10^{-22}$ & $4.6108 \times 10^{-22}$ \\
\hline 250 & $2.9745 \times 10^{-21}$ & $1.0913 \times 10^{-20}$ \\
\hline 500 & $8.0480 \times 10^{-23}$ & 0 \\
\hline 1000 & $-5.3321 \times 10^{-23}$ & $-9.0176 \times 10^{-22}$ \\
\hline
\end{tabular}

Table 2. Mean errors in the synchronization dynamics of the membrane potential states and recovery variable states for different-sized unidirectional networks of FHN neurons without noise.

network-listed in Table 3-show that the proposed control scheme is successful in guaranteeing the synchronization of noisy FHN neuronal networks regardless of the size of the network.

\section{Analysis of bidirectional time-delayed FHN networks.}

\section{(a) Networks without noise}

Similar to the unidirectional network's analysis, we considered bidirectional networks of five, ten, fifty, one hundred, two hundred and fifty, five hundred, and one thousand neurons with different gap-junctions and time delays. The results of this analysis are illustrated in Figs. 6 and 7 and Table 4. The non-synchronized activity of neurons in different bidirectional networks without noise can be visualized in Figs. $6 \mathrm{a}$ and $7 \mathrm{a}$ as the time dynamics of the errors are non-zero and non-convergent for both membrane potential states and recovery variable 


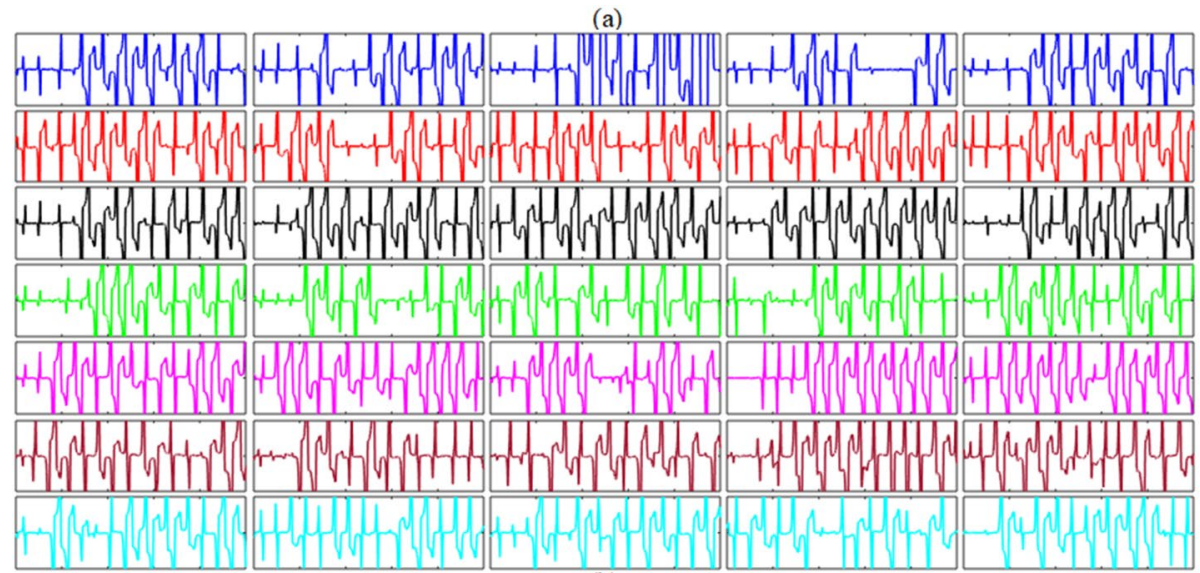

(b)

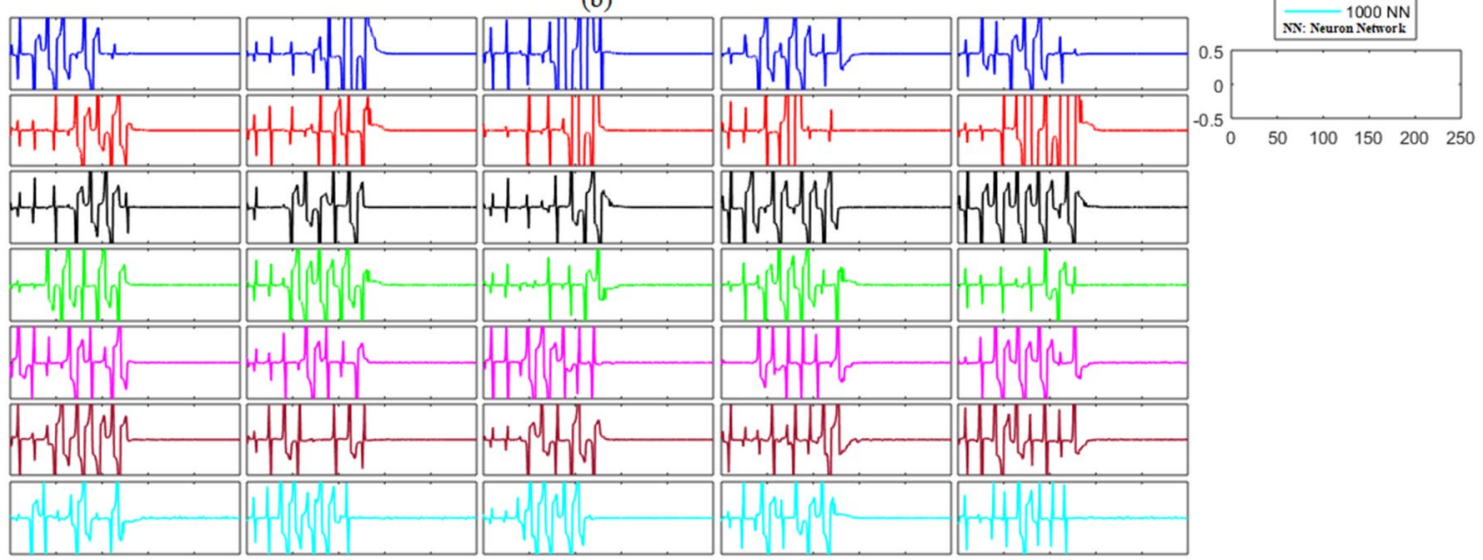

Figure 4. (a) Without control synchronization dynamics of the membrane potential states (x-states) of different time-delayed unidirectional FHN networks with noise. (b) Controlled synchronized error dynamics of the membrane potential states (x-state) of different time-delayed unidirectional FHN networks with noise.

states. Contrastingly, both states of the networks achieved zero error after the application of the proposed control scheme at $t=130$, as shown in Figs. $6 \mathrm{~b}$ and $7 \mathrm{~b}$. The mean errors for both states of each network are listed in Table 4 . These results suggest that each state of the delayed neuronal network achieved synchronized behavior with the state of the corresponding slave neuron because the outcome of the time dynamics of error converged to zero.

\section{(b) Networks with noise}

The dynamical structure of the bidirectional delayed network with noise is more complex and complicated than that of the unidirectional delayed FHN neuronal networks, but the results of the numerical simulations suggest that synchronization can be achieved successfully with the activation of the proposed controller at $t=130$. The time-error dynamics of different-sized networks revealed in Figs. 8a and 9a show the unsynchronized neuronal activities between all neurons for all networks. Next, we analyzed the results after the implementation of the proposed control law for the bidirectional delayed network in Figs. $8 \mathrm{~b}$ and $9 \mathrm{~b}$. These results show the error dynamics before and after the application of the proposed adaptive controller. Similar to the previous cases, the delayed networks showed highly non-synchronized behavior until the proposed controller was switched on. Then, as soon as the controller was applied, all the errors converged to zero, indicating the effectiveness of the proposed scheme. Furthermore, the results listed in Table 5 with very low mean errors also show that different bidirectional networks of noisy time-delayed FHN neurons achieved synchronization, indicating the effectiveness of the proposed scheme.

The convergence of the error dynamics to zero in all cases guaranteed the synchronization of the differentsized ring-structured networks of FHN neurons in the absence and presence of noise with different gap-junctions and time delays under conditions of EES and ionic gate disturbance. It was also observed that the errors between neurons for unidirectional and bidirectional gap-junction networks converged to zero very rapidly in all cases, showing the efficiency of the proposed control schemes. 
(a)

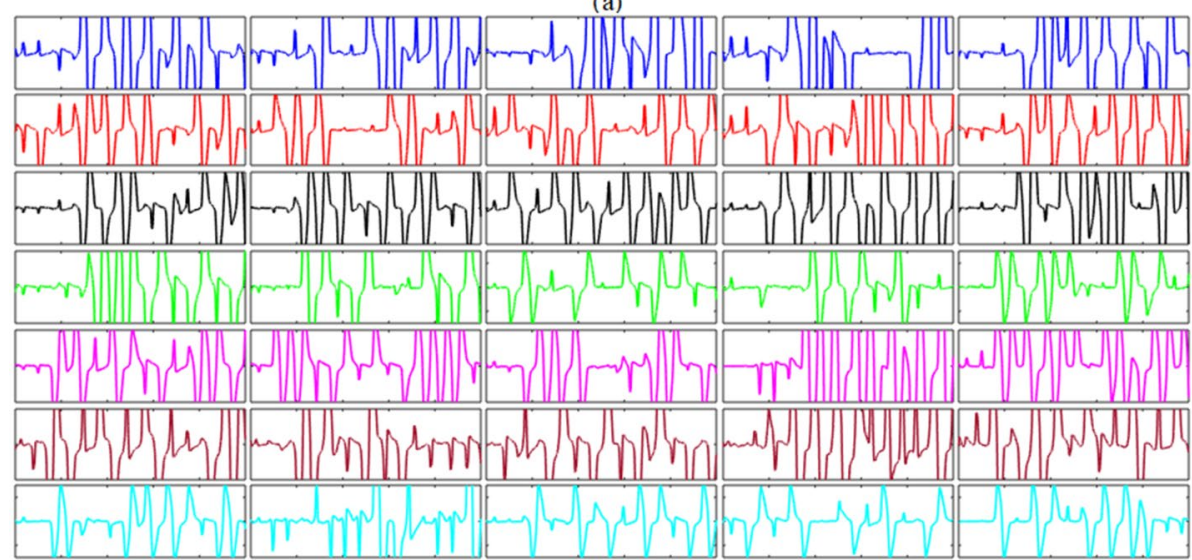

(b)
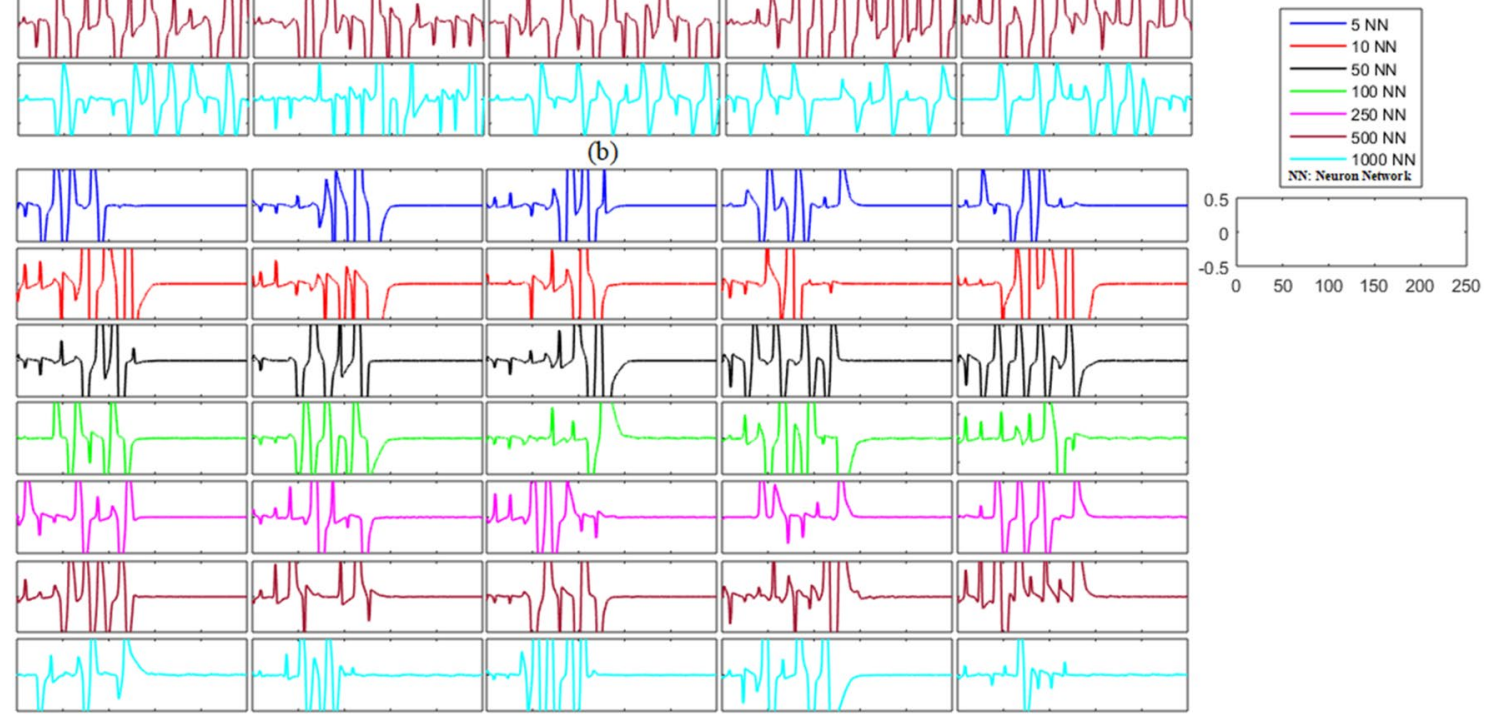

Figure 5. (a) Without control synchronization dynamics of the recovery variable states (y-states) of different time-delayed unidirectional FHN networks with noise. (b) Controlled synchronized error dynamics of the recovery variable states (y-states) of different time-delayed unidirectional FHN neuronal networks with noise.

\begin{tabular}{|l|c|c|}
\hline Number of neurons in network & Membrane potential states & Recovery variable states \\
\hline 5 & $-2.0014 \times 10^{-21}$ & $8.7445 \times 10^{-20}$ \\
\hline 10 & $1.6192 \times 10^{-21}$ & $-2.0768 \times 10^{-20}$ \\
\hline 50 & $4.5356 \times 10^{-22}$ & $-1.5868 \times 10^{-20}$ \\
\hline 100 & $-1.0464 \times 10^{-22}$ & $1.0685 \times 10^{-22}$ \\
\hline 250 & $2.1871 \times 10^{-22}$ & $-1.0531 \times 10^{-21}$ \\
\hline 500 & $-7.8048 \times 10^{-22}$ & $-1.7182 \times 10^{-22}$ \\
\hline 1000 & $5.3821 \times 10^{-24}$ & $-4.8910 \times 10^{-22}$ \\
\hline
\end{tabular}

Table 3. Mean errors in the synchronization dynamics of the membrane potential states and recovery variable states for different-sized unidirectional networks of FHN neurons with noise.

\section{Discussion}

The processing of cognitive information in the brain is based upon the synchronized interactions between large numbers of neurons distributed within and across different specialized brain regions. Experimental and theoretical results from previous studies suggested that synchronization of neuronal activity is not only a fundamental property of cortical and subcortical networks within and across different brain regions but also serves a variety of functions in cognitive processes. It is evident from past research that certain brain disorders, such as Alzheimer's disease, epilepsy, Parkinson's, autism, and schizophrenia are associated with abnormal neural synchronization ${ }^{19}$. Researchers have studied the synchronization problem in neurons by using different mathematical models of neurons ${ }^{71-73}$. Among those, FHN is the most commonly used model to investigate the synchronization of coupled neurons because of its wide applicability and complex dynamical aspects. In the literature, the subject of neuronal synchronization, using the FHN model, has been intensively examined as a potential application in cognitive engineering ${ }^{1,20,28,47,57}$. Researchers have developed adaptive ${ }^{20,41}$, nonlinear ${ }^{28}$, robust control ${ }^{23}$, neuralnetwork-, fuzzy ${ }^{74}$, and observer-based control schemes ${ }^{63}$ to study the synchronization phenomenon in FHN 
(a)
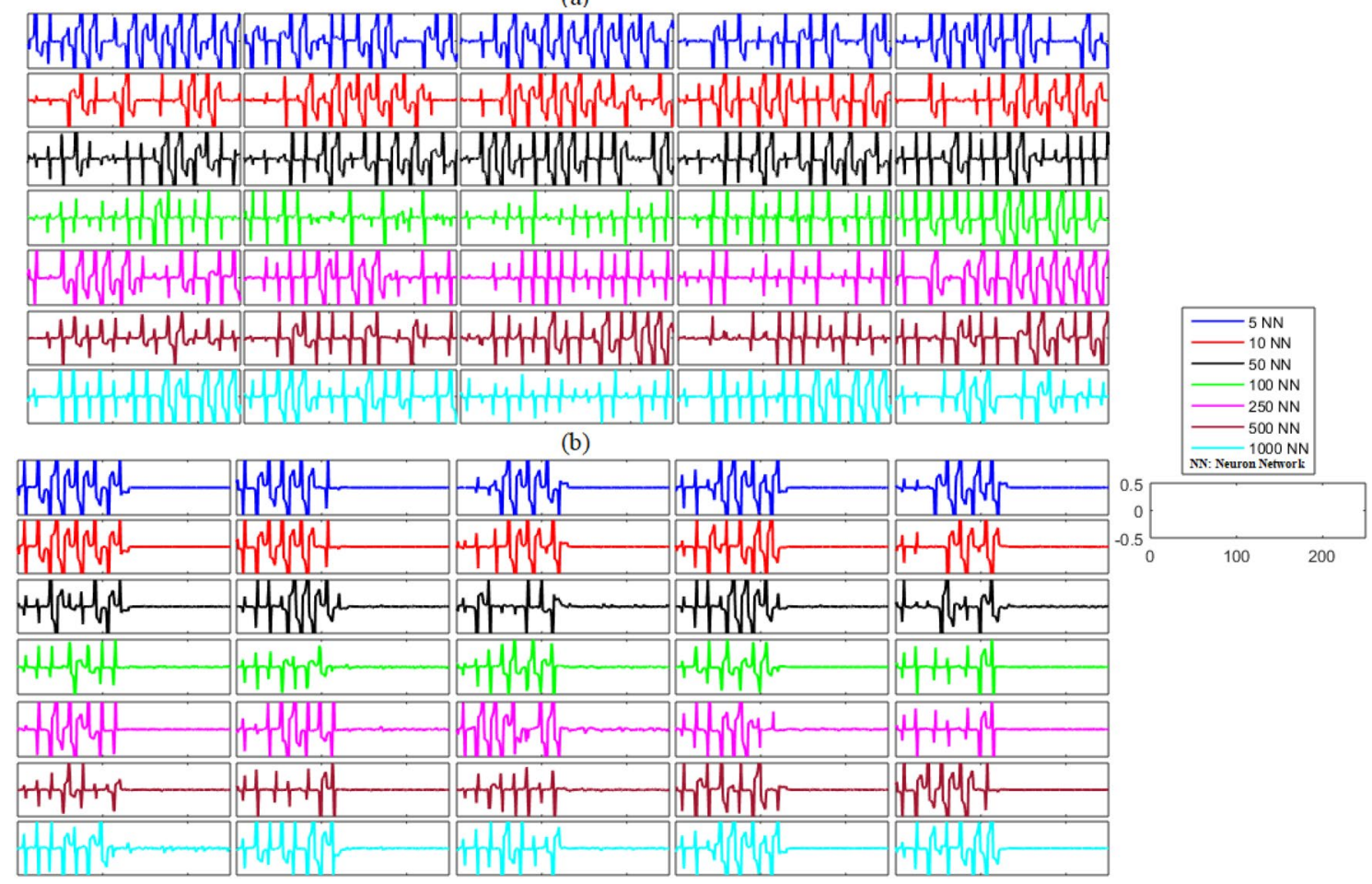

Figure 6. (a) Without control synchronization dynamics of the membrane potential states (x-states) of different time-delayed bidirectional FHN networks without noise. (b) Controlled synchronized error dynamics of the membrane potential states (x-state) of different time-delayed bidirectional FHN networks without noise.

(a)

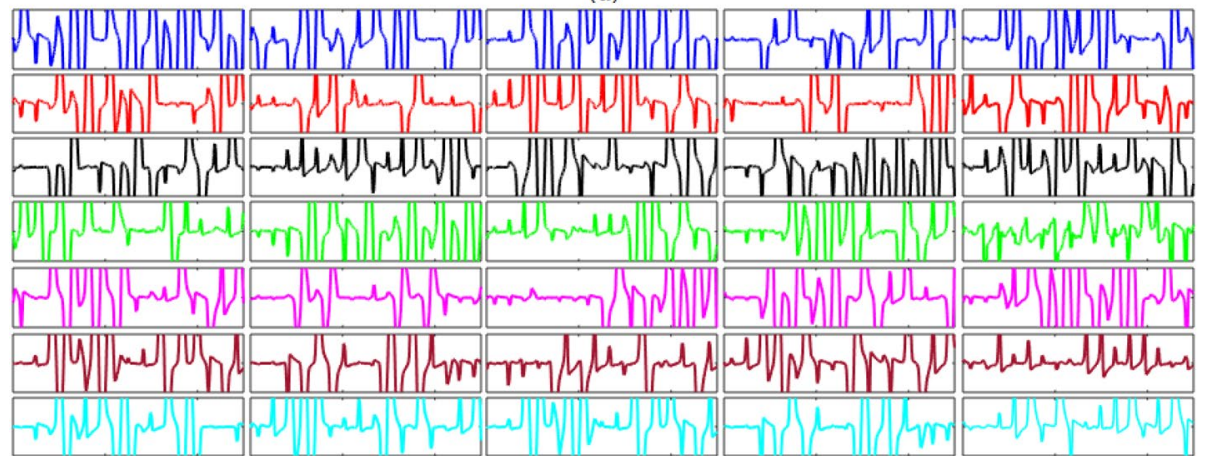

(b)
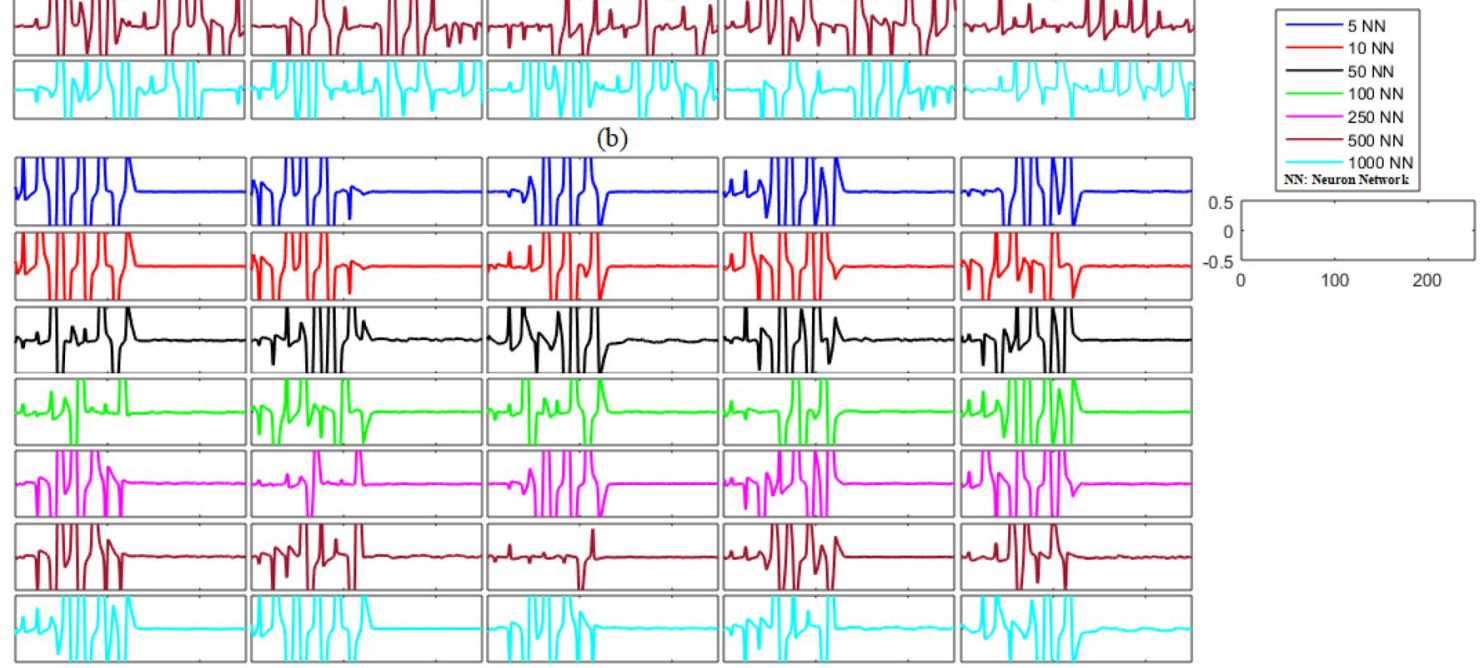

Figure 7. (a) Without control synchronization dynamics of the recovery variable states (y-states) of different time-delayed bidirectional FHN networks without noise. (b) Controlled synchronized error dynamics of the recovery variable states (y-states) of different time-delayed bidirectional FHN neuronal networks without noise. 


\begin{tabular}{|l|c|l|}
\hline Number of neurons in network & Membrane potential states & Recovery variable states \\
\hline 5 & $8.9592 \times 10^{-22}$ & 0 \\
\hline 10 & $5.1245 \times 10^{-20}$ & $1.7956 \times 10^{-19}$ \\
\hline 50 & $-3.8708 \times 10^{-20}$ & $4.8477 \times 10^{-21}$ \\
\hline 100 & $2.6293 \times 10^{-20}$ & $9.3919 \times 10^{-21}$ \\
\hline 250 & $-1.8942 \times 10^{-21}$ & $4.0780 \times 10^{-21}$ \\
\hline 500 & $-3.5844 \times 10^{-21}$ & $2.0709 \times 10^{-20}$ \\
\hline 1000 & $1.5764 \times 10^{-21}$ & $-1.0755 \times 10^{-21}$ \\
\hline
\end{tabular}

Table 4. Mean errors in the synchronization dynamics of the membrane potential states and recovery variable states for different-sized bidirectional networks of FHN neurons without noise.

(a)

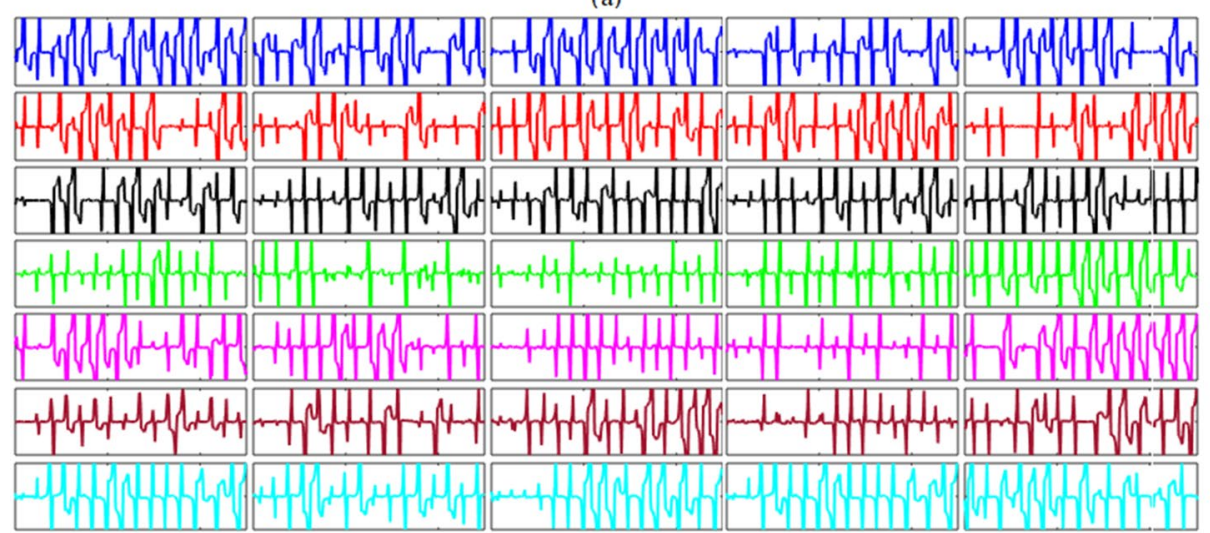

(b)
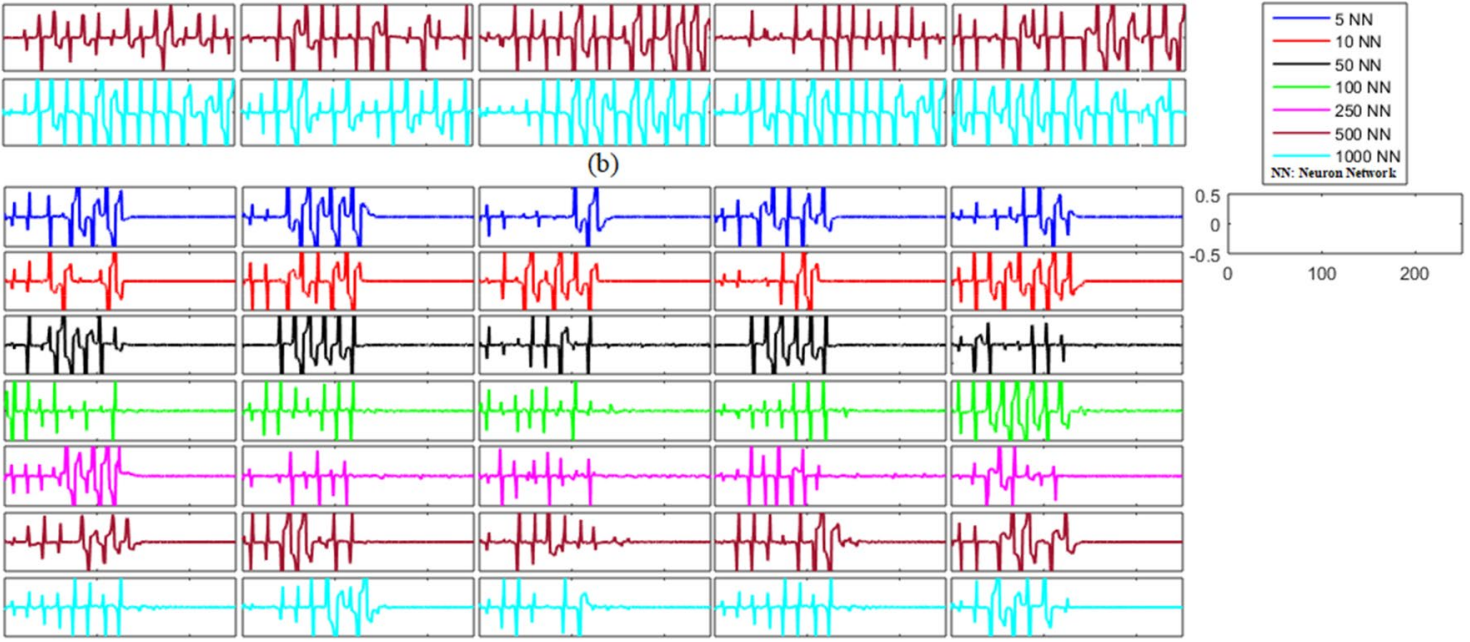

Figure 8. (a) Without control synchronization dynamics of the membrane potential states (x-states) of different time-delayed bidirectional FHN networks with noise. (b) Controlled synchronized error dynamics of the membrane potential states (x-state) of different time-delayed bidirectional FHN networks with noise.

neurons under external electrical stimulations. However, these conventional methodologies were developed for two or three coupled neurons and cannot guarantee synchronization of distant neurons if used for synchronizing the activity of networks of neurons because the mathematical models ignore the time delays arising from the separation between coupled neurons, and hence cannot synchronize distant FHN neurons. Therefore, the addition of a delay term in mathematical models accounting for the distant communication between neurons makes it realistic but more complex to study the synchronization problem. Furthermore, the integration of the gap-junction strength in FHN neurons renders the synchronization dilemma nontrivial ${ }^{75}$. Moreover, previous studies assumed bidirectional coupling between the neurons whereas experimental observation suggests that the coupling between neurons could be unidirectional and may result in different coupling strengths for each neuron $^{55,76}$. Therefore, the dynamical effects of unidirectional gap junctions while entertaining time delays (due to neuronal separation) in the mathematical models should not be ignored. Additionally, it is evident from past research that the presence of noise can affect the dynamical behavior of the neuronal system and neurons adjust their firing properties to transmit information optimally ${ }^{70}$. Therefore, researchers should entertain the noise effect in their mathematical models to study the synchronization problem.

To overcome the shortcomings of previous studies, this study investigated the synchronization problem in the network of coupled FHN neurons by incorporating time delays, different direction-dependent coupling 
(a)

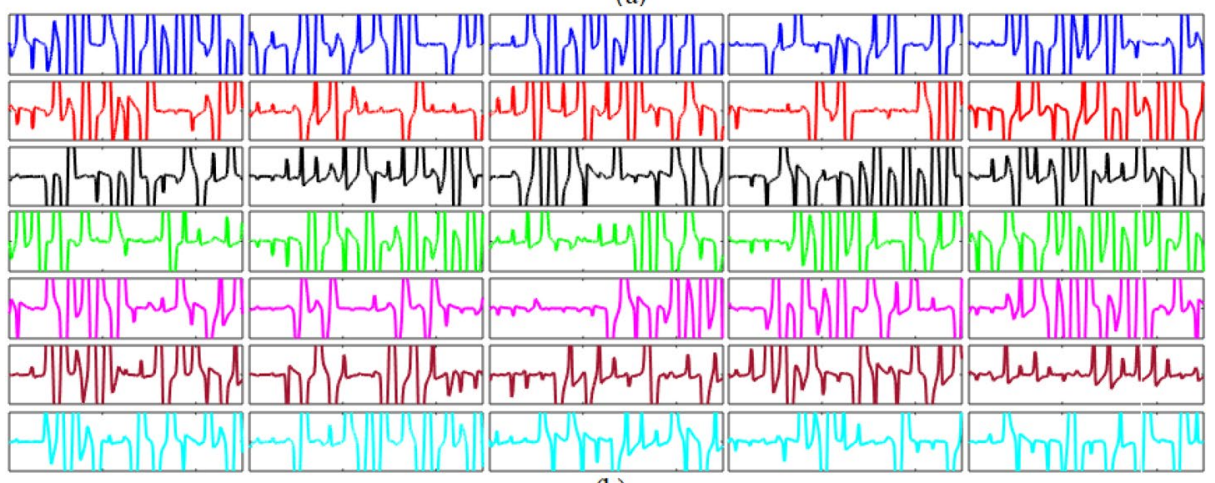

(b)

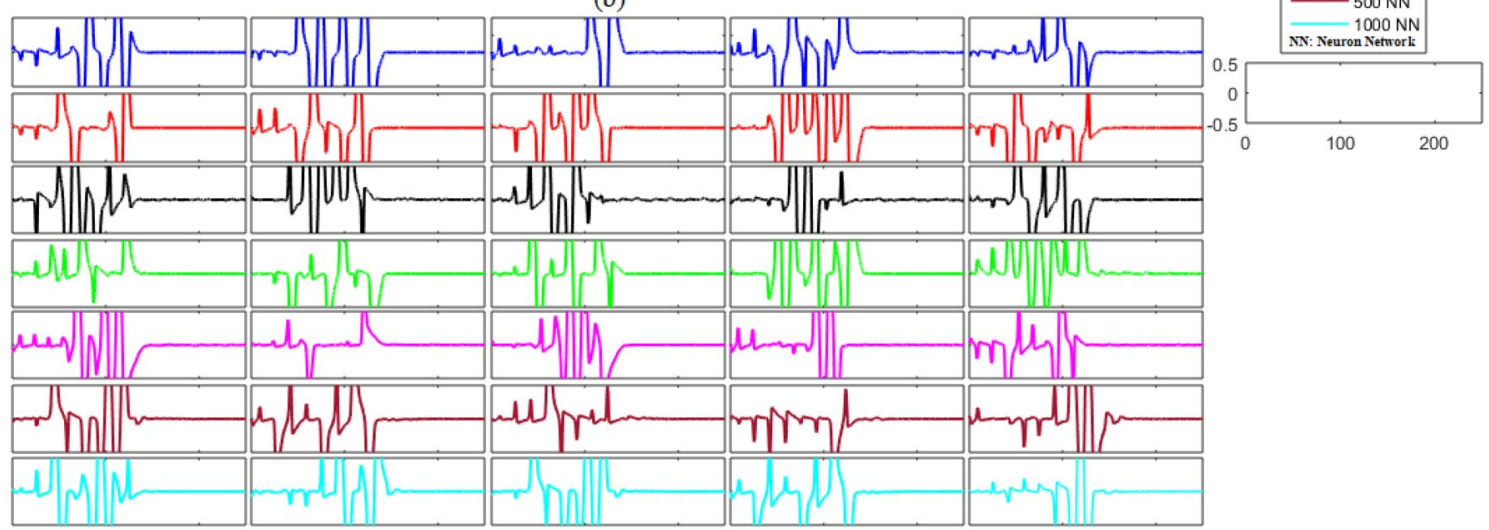

Figure 9. (a) Without control synchronization dynamics of the recovery variable states (y-states) of different time-delayed bidirectional FHN networks with noise. (b) Controlled synchronized error dynamics of the recovery variable states (y-states) of different time-delayed bidirectional FHN neuronal networks with noise.

\begin{tabular}{|l|c|c|}
\hline Number of neurons in network & Membrane potential states & Recovery variable states \\
\hline 5 & $-4.9363 \times 10^{-21}$ & $-1.1989 \times 10^{-20}$ \\
\hline 10 & $-5.0001 \times 10^{-21}$ & $-3.4012 \times 10^{-20}$ \\
\hline 50 & $1.3890 \times 10^{-21}$ & $4.0569 \times 10^{-21}$ \\
\hline 100 & $7.9239 \times 10^{-22}$ & $2.6784 \times 10^{-21}$ \\
\hline 250 & $-5.9572 \times 10^{-22}$ & $-1.0239 \times 10^{-22}$ \\
\hline 500 & $-1.2124 \times 10^{-21}$ & $-5.6866 \times 10^{-22}$ \\
\hline 1000 & $-2.7764 \times 10^{-21}$ & $2.0092 \times 10^{-21}$ \\
\hline
\end{tabular}

Table 5. Mean errors in the synchronization dynamics of the membrane potential states and recovery variable states for different-sized bidirectional networks of FHN neurons with noise.

(unidirectional and bidirectional), noise, and ionic and external disturbances in mathematical models. In contrast to traditional techniques, we proposed simple adaptive control schemes that guarantee the synchronization of unidirectional and bidirectional time-delayed networks of FHN neurons in the absence and presence of noise. The efficacy of the proposed control laws is shown through numerical simulations of different-sized networks consisting of five, ten, fifty, one hundred, two hundred and fifty, five hundred, and one thousand neurons. The results presented in Figs. 2, 3, 4, 5, 6, 7, 8 and 9 and Tables 2, 3, 4 and 5 validate the efficiency of the proposed control laws.

Although the present study proposed efficient control schemes that guarantee the synchronization of networks of time-delayed FHN neurons, it also has some limitations/drawbacks. One obvious of this study is that we considered the networks of identical FHN neurons with known fixed parameters in the present configurations. However, the possibility of two coupled neurons to be non-identical cannot be ignored, and the actual model parameters cannot be completely known, owing to the known dynamics of the brain and biological restrictions. Therefore, considering a network of large numbers of non-identical neurons having unknown parametric values where several hundred neurons communicate with each other under different directiondependent couplings (unidirectional and bidirectional), can enhance the complexity and analysis of neuronal synchronization. The understanding of the physical theory behind direction-dependent couplings, it's discrete 
modeling, and simulations is a complex and tedious job; hence, it is a challenging task for the research community. Thus, it is our immediate future plan to study the synchronization of the network of non-identical FHN neurons with unknown parameters and disturbances. Moreover, in the current configurations, the coupling between the neurons of each network is assumed to be unidirectional or bidirectional, but in a real scenario, a network may have both types of direction-dependent coupling that will enhance the complexity of the network. Another possible future direction of the current study is that we could investigate the link between mathematical simulations and experimental data recorded from real neurons, as this could help to understand the dynamics of various brain disorders ${ }^{19}$. This could be done by estimating the parameters of FHN neurons to replicate the experimental data. For instance, Che et al. $^{77}$ developed an identification method to estimate the parameters of FHN models to replicate the experimental data recorded from real neurons. Furthermore, in the present study, only synchronization within a network of neurons is considered and how two or more networks with different dynamics and configurations (non-identical neurons, unknown parameters, external disturbances, and different direction-dependent coupling) communicate and synchronize their activities is yet to be explored in future work.

\section{Conclusion}

The development of control strategies for synchronization of a network of chaotic neurons with time delays, different direction-dependent coupling (unidirectional and bidirectional), and noise, particularly under external disturbances, is essential and very challenging. Most of the previous studies developed control strategies for two or three coupled neurons with bidirectional coupling and without incorporating the effect of noise, but not for time-delayed neural networks. To overcome these limitations, this study investigated the synchronization problem in the network of coupled FHN neurons by incorporating time delays, different direction-dependent coupling (unidirectional and bidirectional), noise, and ionic and external disturbances in mathematical models. Both unidirectional and bidirectional time-delayed FHN neuronal networks have very complex and unpredictable behavior and dynamics. In this study, the lag synchronization of a network of delayed FHN neurons with unidirectional and bidirectional coupling in the absence and presence of noise was addressed. Different gap junctions and time-delay parameters were used to incorporate the dynamics of time delays in neurons. Two different networks, one with unidirectional coupling between two neurons and the other with bidirectional coupling in membrane states, were considered. To achieve the synchronization between the states of the delayed neuronal networks, we designed two different adaptive control strategies, which compensated for the nonlinear dynamics without direct cancelation. Lyapunov stability theory was used to derive sufficient conditions that guarantee the synchronization of the delayed FHN neuronal networks. Numerical simulations with networks of five, ten, fifty, one hundred, two hundred and fifty, five hundred, and one thousand neurons were performed to demonstrate the efficiency of the proposed control schemes.

Received: 9 October 2020; Accepted: 27 January 2021

Published online: 16 February 2021

\section{References}

1. Ibrahim, M. M. \& Jung, I. H. Complex synchronization of a ring-structured network of FitzHugh-Nagumo neurons with singleand dual-state gap junctions under ionic gates and external electrical disturbance. IEEE Access 7, 57894-57906 (2019).

2. Li, C., Liao, X. \& Wong, K.-W. Chaotic lag synchronization of coupled time-delayed systems and its applications in secure communication. Physica D 194, 187-202 (2004).

3. Bao, H. \& Cao, J. Finite-time generalized synchronization of nonidentical delayed chaotic systems. Nonlinear Anal. Modell. Control 21, 306-324 (2016).

4. Rosenblum, M. G., Pikovsky, A. S. \& Kurths, J. Phase synchronization of chaotic oscillators. Phys. Rev. Lett. 76, 1804 (1996).

5. Cun-Fang, F., Yan, Z. \& Ying-Hai, W. Projective synchronization in time-delayed chaotic systems. Chin. Phys. Lett. 23, 1418 (2006).

6. Han, Q., Li, C. \& Huang, J. Anticipating synchronization of chaotic systems with time delay and parameter mismatch. Chaos Interdiscip. J. Nonlinear Sci. 19, 013104 (2009).

7. Della Rossa, F. et al. Symmetries and cluster synchronization in multilayer networks. Nat. Commun. 11, 1-17 (2020).

8. Kazemy, A. \& Cao, J. Consecutive synchronization of a delayed complex dynamical network via distributed adaptive control approach. Int. J. Control Autom. Syst. 16, 2656-2664 (2018).

9. Mannan, M. M. N., Jeong, M. Y. \& Kamran, M. A. Hybrid ICA-Regression: Automatic identification and removal of ocular artifacts from electroencephalographic signals. Front. Hum. Neurosci. 10, 193 (2016).

10. Al-Shargie, F., Tang, T. B. \& Kiguchi, M. Stress assessment based on decision fusion of EEG and fNIRS signals. IEEE Access 5, 19889-19896 (2017).

11. Wang, Y., Cai, L., Luo, X., Ying, W. \& Gao, H. simulation of action potential propagation based on the ghost structure method. Sci. Rep. 9, 1-18 (2019).

12. Mannan, M., Kim, S., Jeong, M. \& Kamran, M. Hybrid EEG-Eye tracker: Automatic identification and removal of eye movement and blink artifacts from electroencephalographic signal. Sensors 16, 241 (2016).

13. Lin, B.-S., Chen, J.-L. \& Hsu, H.-C. Novel upper-limb rehabilitation system based on attention technology for post-stroke patients: A preliminary study. IEEE Access 6, 2720-2731 (2017).

14. Kamran, M. A., Jeong, M. Y. \& Mannan, M. Optimal hemodynamic response model for functional near-infrared spectroscopy. Front. Behav. Neurosci. 9, 151 (2015).

15. Kamran, M. A., Mannan, M. M. N. \& Jeong, M. Y. Cortical signal analysis and advances in functional near-infrared spectroscopy signal: A review. Front. Hum. Neurosci. 10,261 (2016).

16. Zafar, R. et al. Prediction of human brain activity using likelihood ratio based score fusion. IEEE Access 5, 13010-13019 (2017).

17. Mannan, M. M. N., Kamran, M. A., Kang, S. \& Jeong, M. Y. Effect of EOG signal filtering on the removal of ocular artifacts and EEG-based brain-computer interface: A comprehensive study. Complexity 2018 (2018).

18. Wang, H., Lei, X., Zhan, Z., Yao, L. \& Wu, X. A new fMRI informed mixed-norm constrained algorithm for EEG source localization. IEEE Access 6, 8258-8269 (2018).

19. Uhlhaas, P. J. \& Singer, W. Neural synchrony in brain disorders: Relevance for cognitive dysfunctions and pathophysiology. Neuron 52, 155-168 (2006). 
20. Yu, H. et al. Adaptive backstepping sliding mode control for chaos synchronization of two coupled neurons in the external electrical stimulation. Commun. Nonlinear Sci. Numer. Simul. 17, 1344-1354 (2012).

21. Singer, W. Synchronization of cortical activity and its putative role in information processing and learning. Annu. Rev. Physiol. 55, 349-374 (1993).

22. Sun, X. \& Li, G. Synchronization transitions induced by partial time delay in a excitatory-inhibitory coupled neuronal network. Nonlinear Dyn. 89, 2509-2520 (2017).

23. Rigatos, G. Robust synchronization of coupled neural oscillators using the derivative-free nonlinear Kalman Filter. Cogn. Neurodyn. 8, 465-478 (2014).

24. Freund, H.-J. Motor unit and muscle activity in voluntary motor control. Physiol. Rev. 63, 387-436 (1983).

25. Levy, R., Hutchison, W. D., Lozano, A. M. \& Dostrovsky, J. O. High-frequency synchronization of neuronal activity in the subthalamic nucleus of parkinsonian patients with limb tremor. J. Neurosci. 20, 7766-7775 (2000).

26. Popovych, O. V. \& Tass, P. A. Adaptive delivery of continuous and delayed feedback deep brain stimulation-A computational study. Sci. Rep. 9, 10585 (2019).

27. Shahal, S. et al. Synchronization of complex human networks. Nat. Commun. 11, 1-10 (2020).

28. Wang, J., Deng, B. \& Fei, X. Chaotic synchronization of two coupled neurons via nonlinear control in external electrical stimulation. Chaos Solitons Fractals 27, 1272-1278 (2006).

29. Joseph, G. V. \& Pakrashi, V. Limits on anti-phase synchronization in oscillator networks. Sci. Rep. 10, 1-9 (2020).

30. Ziaeemehr, A., Zarei, M. \& Sheshbolouki, A. Emergence of global synchronization in directed excitatory networks of type I neurons. Sci. Rep. 10, 1-11 (2020).

31. Bennett, M. \& Verselis, V. in Seminars in Cell Biology. 29-47 (Elsevier, Amsterdam).

32. Izhikevich, E. M. Which model to use for cortical spiking neurons?. IEEE Trans. Neural Netw. 15, 1063-1070 (2004).

33. Hodgkin, A. L. \& Huxley, A. F. A quantitative description of membrane current and its application to conduction and excitation in nerve. J. Physiol. 117, 500 (1952).

34. Morris, C. \& Lecar, H. Voltage oscillations in the barnacle giant muscle fiber. Biophys. J. 35, 193-213 (1981).

35. Hindmarsh, J. L. \& Rose, R. A model of neuronal bursting using three coupled first order differential equations. Proc. R. Soc. Lond. Ser. B. Biol. Sci. 221, 87-102 (1984).

36. Fitzhugh, R. Thresholds and plateaus in the Hodgkin-Huxley nerve equations. J. Gen. Physiol. 43, 867-896 (1960).

37. Nagumo, J., Arimoto, S. \& Yoshizawa, S. An active pulse transmission line simulating nerve axon. Proc. IRE 50, 2061-2070 (1962).

38. Masoliver, M. \& Masoller, C. Sub-threshold signal encoding in coupled FitzHugh-Nagumo neurons. Sci. Rep. 8, 1-10 (2018).

39. Mondal, A., Sharma, S. K., Upadhyay, R. K. \& Mondal, A. Firing activities of a fractional-order FitzHugh-Rinzel bursting neuron model and its coupled dynamics. Sci. Rep. 9, 1-11 (2019).

40. Wang, C. \& Ge, S. S. Synchronization of two uncertain chaotic systems via adaptive backstepping. Int. J. Bifurc. Chaos 11, 1743-1751 (2001).

41. Wang, Y., Zhang, X., Yang, L. \& Huang, H. Adaptive synchronization of time delay chaotic systems with uncertain and unknown parameters via aperiodically intermittent control. Int. J. Control Autom. Syst. 18, 696-707 (2020).

42. Schöll, E., Hiller, G., Hövel, P. \& Dahlem, M. A. Time-delayed feedback in neurosystems. Philos. Trans. R. Soc. A Math. Phys. Eng. Sci. 367, 1079-1096 (2009).

43. Chen, Y.-S., Hwang, R. R. \& Chang, C.-C. Adaptive impulsive synchronization of uncertain chaotic systems. Phys. Lett. A 374, 2254-2258 (2010).

44. Yu, H., Wang, J., Liu, C., Deng, B. \& Wei, X. Delay-induced synchronization transitions in modular scale-free neuronal networks with hybrid electrical and chemical synapses. Phys. A 405, 25-34 (2014).

45. Arenas, A., Díaz-Guilera, A., Kurths, J., Moreno, Y. \& Zhou, C. Synchronization in complex networks. Phys. Rep. 469, 93-153 (2008).

46. Ouannas, A., Wang, X., Pham, V.-T. \& Ziar, T. Dynamic analysis of complex synchronization schemes between integer order and fractional order chaotic systems with different dimensions. Complexity 2017 (2017).

47. Mehdiabadi, M. R., Rouhani, E., Mashhadi, S. M. \& Jalali, A. Adaptive fractional-order control for synchronization of two coupled neurons in the external electrical stimulation. Basic Clin. Neurosci. 5, 144 (2014).

48. Nitsan, I., Drori, S., Lewis, Y. E., Cohen, S. \& Tzlil, S. Mechanical communication in cardiac cell synchronized beating. Nat. Phys. 12, $472(2016)$

49. Ziaeemehr, A., Zarei, M., Valizadeh, A. \& Mirasso, C. R. Frequency-dependent organization of the brain's functional network through delayed-interactions. Neural Netw. 132, 155-165 (2020).

50. Pariz, A. et al. High frequency neurons determine effective connectivity in neuronal networks. NeuroImage 166, 349-359 (2018).

51. Esfahani, Z. G., Gollo, L. L. \& Valizadeh, A. Stimulus-dependent synchronization in delayed-coupled neuronal networks. Sci. Rep. 6, 1-10 (2016)

52. Esfahani, Z. G. \& Valizadeh, A. Zero-lag synchronization despite inhomogeneities in a relay system. PLoS ONE 9, e112688 (2014).

53. Wang, Q., Perc, M., Duan, Z. \& Chen, G. Synchronization transitions on scale-free neuronal networks due to finite information transmission delays. Phys. Rev. E 80, 026206 (2009).

54. Tang, J., Ma, J., Yi, M., Xia, H. \& Yang, X. Delay and diversity-induced synchronization transitions in a small-world neuronal network. Phys. Rev. E 83, 046207 (2011).

55. Dhamala, M., Jirsa, V. K. \& Ding, M. Enhancement of neural synchrony by time delay. Phys. Rev. Lett. 92, 074104 (2004).

56. Yang, X., Li, H. \& Sun, Z. Partial coupling delay induced multiple spatiotemporal orders in a modular neuronal network. PLoS ONE 12, e0177918 (2017).

57. Zhen, B., Li, Z. \& Song, Z. Influence of time delay in signal transmission on synchronization between two coupled FitzHughNagumo neurons. Appl. Sci. 9, 2159 (2019).

58. Jia, J., Liu, H., Xu, C. \& Yan, F. Dynamic effects of time delay on a coupled FitzHugh-Nagumo neural system. Alexandria Eng. J. 54, 241-250 (2015).

59. Zhang, W., Cao, J., Chen, D. \& Alsaedi, A. Out lag synchronization of fractional order delayed complex networks with coupling delay via pinning control. Complexity 2019 (2019).

60. Petkoski, S. \& Jirsa, V. K. Transmission time delays organize the brain network synchronization. Philos. Trans. R. Soc. A 377, $20180132(2019)$

61. 61Liu, H. \& Zhang, P. Phase synchronization dynamics of neural network during seizures. Comput. Math. Methods Med. 2018 (2018).

62. Siddique, M., Rehan, M., Bhatti, M. \& Ahmed, S. Delay-range-dependent local adaptive and robust adaptive synchronization approaches for time-delay chaotic systems. Nonlinear Dyn. 88, 2671-2691 (2017).

63. Siddique, M. \& Rehan, M. A concept of coupled chaotic synchronous observers for nonlinear and adaptive observers-based chaos synchronization. Nonlinear Dyn. 84, 2251-2272 (2016).

64. Riaz, M., Rehan, M. \& Ashraf, M. Synchronization of nonlinear master-slave systems under input delay and slope-restricted input nonlinearity. Complexity 21, 220-233 (2016).

65. Zaheer, M. H., Rehan, M., Mustafa, G. \& Ashraf, M. Delay-range-dependent chaos synchronization approach under varying timelags and delayed nonlinear coupling. ISA Trans. 53, 1716-1730 (2014). 
66. 66Rehan, M. \& Hong, K.-S. Robust synchronization of delayed chaotic FitzHugh-Nagumo neurons under external electrical stimulation. Comput. Math. Methods Med. 2012 (2012).

67. Wellens, T., Shatokhin, V. \& Buchleitner, A. Stochastic resonance [02.50. Ey Stochastic processes; 05.40. Ca Noise; 32.80.-t Photon interactions with atoms; 42.50. Lc Quantum fluctuations, quantum noise, and quantum jumps; 87.10.+ e General theory and mathematical aspects;]. (2004).

68. Pikovsky, A. S. \& Kurths, J. Coherence resonance in a noise-driven excitable system. Phys. Rev. Lett. 78, 775 (1997).

69. Tessone, C. J., Scire, A., Toral, R. \& Colet, P. Theory of collective firing induced by noise or diversity in excitable media. Phys. Rev. E 75, 016203 (2007)

70. Acebrón, J., Bulsara, A. \& Rappel, W.-J. Noisy FitzHugh-Nagumo model: From single elements to globally coupled networks. Phys. Rev. E 69, 026202 (2004).

71. Wang, G., Jin, W. \& Wang, A. The complete synchronization of coupled Morris-Lecar neurons with chemical synapses. Int. J. Mod. Phys. B 30, 1650096 (2016).

72. Aguilar-López, R. \& Martínez-Guerra, R. Synchronization of a coupled Hodgkin-Huxley neurons via high order sliding-mode feedback. Chaos Solitons Fractals 37, 539-546 (2008).

73. Yu, H. \& Peng, J. Chaotic synchronization and control in nonlinear-coupled Hindmarsh-Rose neural systems. Chaos Solitons Fractals 29, 342-348 (2006).

74. Wang, J., Zhang, Z. \& Li, H. Synchronization of FitzHugh-Nagumo systems in EES via H $\infty$ variable universe adaptive fuzzy control. Chaos Solitons Fractals 36, 1332-1339 (2008).

75. Iqbal, M., Rehan, M., Khaliq, A., Rehman, S.-u. \& Hong, K.-S. Synchronization of coupled different chaotic FitzHugh-Nagumo neurons with unknown parameters under communication-direction-dependent coupling. Comput. Math. Methods Med. 2014 (2014).

76. Horikawa, Y. Exponential transient propagating oscillations in a ring of spiking neurons with unidirectional slow inhibitory synaptic coupling. J. Theor. Biol. 289, 151-159 (2011).

77. Che, Y., Geng, L.-H., Han, C., Cui, S. \& Wang, J. Parameter estimation of the FitzHugh-Nagumo model using noisy measurements for membrane potential. Chaos Interdiscip. J. Nonlinear Sci. 22, 023139 (2012).

\title{
Acknowledgements
}

This research was supported by the Basic Science Research Program through the National Research Foundation of Korea (NRF) funded by the Ministry of Science, ICT and Future Planning (NRF-2017R1E1A1A03070224; NRF-2019R1A2C2007249). This research was supported by the National Research Foundation of Korea (NRF) grants funded by the Korea government (MSIT) (NRF-2017R1A5A1015722).

\section{Author contributions}

MMI contributed to conceptualize, design, implement, draft, critically revise, and approve the final version of the manuscript. MAK and MMNM contributed to implement, draft, critically revise, and approve the final version of the manuscript. SK and IJ contributed to conceptualize, critically revise, and approve the final version of the manuscript.

\section{Competing interests}

The authors declare no competing interests.

\section{Additional information}

Supplementary Information The online version contains supplementary material available at (https://doi. org/10.1038/s41598-021-82886-x).

Correspondence and requests for materials should be addressed to I.H.J. or S.K.

Reprints and permissions information is available at www.nature.com/reprints.

Publisher's note Springer Nature remains neutral with regard to jurisdictional claims in published maps and institutional affiliations.

\begin{abstract}
Open Access This article is licensed under a Creative Commons Attribution 4.0 International License, which permits use, sharing, adaptation, distribution and reproduction in any medium or format, as long as you give appropriate credit to the original author(s) and the source, provide a link to the Creative Commons licence, and indicate if changes were made. The images or other third party material in this article are included in the article's Creative Commons licence, unless indicated otherwise in a credit line to the material. If material is not included in the article's Creative Commons licence and your intended use is not permitted by statutory regulation or exceeds the permitted use, you will need to obtain permission directly from the copyright holder. To view a copy of this licence, visit http://creativecommons.org/licenses/by/4.0/.
\end{abstract}

(C) The Author(s) 2021 